\section{Ultrasound-guided subcostal-posterior transversus abdominis plane block for pain control following laparoscopic sleeve gastrectomy}

\section{To the Editor}

I have read with great interest the article "Ultrasoundguided subcostal-posterior transversus abdominis plane (TAP) block for pain control following laparoscopic sleeve gastrectomy". ${ }^{1}$

Firstly, I would like to bow my head out of respect to the great "Al Fatih Sultan Mohammad" in whose honor their Educational and Research Hospital was named.

Secondly, I would like to congratulate the researchers for concluding that subcostal and posterior TAP block combined provided equivalent analgesia to subcostal TAP block alone, following laparoscopic sleeve gastrectomy. Such an outcome avoids the cumbersome and time consuming "combined" procedure in obese patients, ${ }^{2}$ which may sometimes disturb the timing and scheduling of other operations in a busy operating theater.

The posterior TAP block was initially performed for its effectiveness in lower abdominal procedures. Choosing the method of anesthetic block is mainly determined by the type of abdominal surgery. Therefore, it is not surprising that Albrecht et $\mathrm{al}^{3}$ found that bilateral TAP block did not provide additional analgesic benefit when combined with local anesthetic infiltration of the trocar insertion site for laparoscopic gastric bypass surgery.

Methodologically speaking, the authors mentioned that 'all surgeries were performed by the same surgeon", but most importantly, it is not clear whether the procedures under study, namely subcostal and posterior TAP block, were treated the same. It is not also clear from their intervention description in the Methods section whether the block was performed before or immediately after surgery.

I am not a statistician and therefore I cannot comment on whether it was feasible to perform a pilot study to determine the proper sample size for their study. Most studies mentioned in their discussion, namely, Sinha et al, ${ }^{2}$ Albrecht et $a l,{ }^{3}$ and Ortiz et al, ${ }^{4}$ have enrolled almost twice the number of authors' sample size. In my opinion, a pilot study can determine the minimum valid sample size but a reasonably higher sample size confers a more reliable results and a greater validity of the research inference.
Taking into consideration that obese patients may require higher local anesthetic doses compared with non-obese patients, ${ }^{5}$ it is not know whether the result the authors achieved is due to genuine indifference between the subcutaneous and posterior planes or due to inadequate local anesthetic quantity for the larger TAP in obese patients. The authors' comments on their study limitations have clearly addressed the requirement for further studies with larger sample size that are focused on determining optimum effective dose and volume of local anesthetic.

Finally, it appears from the authors' conclusion as well as from Lee et $\mathrm{al}^{6}$ and Bhatia et $\mathrm{al}^{7}$ conclusions, that "a consistent block above T10 cannot be expected following ultrasound-guided posterior TAP block which is better reserved for abdominal surgery with infra-umbilical incisions", ${ }^{6}$ and for surgeries with mainly supra-umbilical incisions, "subcostal TAP block may be a better alternative than the posterior approach for providing postoperative analgesia".

\section{Abdulla Alabassi Department of Surgery \\ Saqr Hospital \& Medical \& Health Sciences University Ras Al-Khaiman, United Arab Emirates}

\section{Reply from the Author}

We would thank Dr. Abdulla Alabassi for his interest in our study and honoring Fatih Sultan Mehmet in the name of our hospital.

In our study, transversus abdominis plane (TAP) block was performed by the same anesthetist in the recovery room in order to prevent the disturbance of the operating room schedule. The surgeon applied sleeve gastrectomy with the same technique in all patients. It is suggested that posterior TAP block is effective only for low abdominal operations and that upper operation area requires subcostal approach. However, Sinha et al ${ }^{2}$ obtained satisfying analgesia with posterior TAP block in laparoscopic gastrectomy patients while Albreich et $\mathrm{al}^{3}$ did not find any additional beneficial effects of subcostal TAP block when combined with local anesthetic infiltration of the trocar insertion site. The results of these 2 studies conducted us to investigate the advantage of the combination of both techniques.

Although it is considered that obese patients may require higher local anesthetic doses compared with non-obese patients, we could not show any additional benefice of the combination of posterior and subcostal approaches. The greatest analgesic effect of the subcostal block was in the region covering the surgical area, which 
could explain the lack of additional analgesic effect of a posterior TAP block.

According to the pilot study, the power analysis determined that minimum 19 patients were required in each group. To account for the possibility of lack of data collection and exclusion from the study, 20 patients were included in each group. We addressed the small sample size as a limitation of our study and indicated the requirement for further studies with larger sample size that are focused on determining optimum effective dose and volume of local anesthetic.

Dilek E. Arı Department of Anesthesiology and Reanimation Fatih Sultan Mehmet Educational and Research Hospital Istanbul, Turkey ORCID ID: 0000-0002-3734-5323

\section{References}

1. Arı DE, Ar AY, Karip CS, Köksal C, Aydın MT, Gazi M, et al. Ultrasound-guided subcostal-posterior transversus abdominis plane (TAP) block for pain control following laparoscopic sleeve gastrectomy. Saudi Med J 2017; 38: 1224-1229.
2. Sinha A, Jayaraman L, Punhani D. Efficacy of ultrasoundguided transversus abdominis plane block after laparoscopic bariatric surgery: a double blind, randomized, controlled study. Obes Surg 2013; 23: 548-553.

3. Albrecht E, Kirkham KR, Endersby RV, Chan VW, Jackson T, Okrainec A, et al. Ultrasound-guided transversus abdominis plane (TAP) block for laparoscopic gastric-bypass surgery: a prospective randomized controlled double-blind trial. Obes Surg 2013; 23: 1309-1314.

4. Ortiz J, Suliburk JW, Wu K, Bailard NS, Mason C, Minard $\mathrm{CG}$, et al. Bilateral transverses abdominis plane block does not decrease postoperative pain after laparoscopic cholecystectomy when compared with local anesthetic infiltration of trocar insertion sites. Reg Anesth Pain Med 2012; 37: 188-192.

5. Gravante G, Castri F, Araco F, Araco A. A Comparative study of the transversus abdominis plane (TAP) block efficacy on postbariatric vs aesthetic abdominoplasty with flank liposuction. Obes Surg 2011; 21: 278-282.

6. Lee TH, Barrington MJ, Tran TM, Wong D, Hebbard PD. Comparison of extent of sensory block following posterior and subcostal approaches to ultrasound-guided transverses abdominis plane block. Anesth Intensive Care 2010; 38: 452-460.

7. Bhatia N, Arora S, Wig J, Kaur G. Comparison of posterior and subcostal approaches to ultrasound-guided transverses abdominis plane block for postoperative analgesia in laparoscopic cholecystectomy. J Clin Anesth 2014; 26: 294-299. 